\title{
Delay of phagosome maturation by a mycobacterial lipid is reversed by nitric oxide
}

Sofia Axelrod, ${ }^{1 \dagger}$ Hartmut Oschkinat, ${ }^{2}$ Jana Enders, ${ }^{1}$ Brigitte Schlegel, ${ }^{2}$ Volker Brinkmann, ${ }^{3}$

Stefan H. E. Kaufmann, ${ }^{1}$ Albert Haas ${ }^{4}$ and

Ulrich E. Schaible ${ }^{1,5 *}$

${ }^{1}$ Department of Immunology, Max Planck Institute for Infection Biology, D-10117 Berlin, Germany.

${ }^{2}$ Leibniz-Institut für Molekulare Pharmakologie, D-13125

Berlin-Buch, Germany.

${ }^{3}$ Microscopy Core Facility, Max Planck Institute for

Infection Biology, D-10117 Berlin, Germany.

${ }^{4}$ Institute for Cell Biology, University Bonn, D-53121

Bonn, Germany.

${ }^{5}$ London School of Hygiene and Tropical Medicine, Infectious and Tropical Diseases-Immunology; WC1E 7HT, London, UK.

\section{Summary}

Mycobacterium tuberculosis is a facultative intracellular pathogen that inhibits phagosome maturation in macrophages thereby securing survival and growth. Mycobacteria reside in an early endocytic compartment of near-neutral $\mathrm{pH}$ where they upregulate production of complex glycolipids such as trehalose dimycolate. Here, we report that trehalose dimycolate coated onto beads increased the bead retention in early phagosomes, i.e. at a similar stage as viable mycobacteria. Thus, a single mycobacterial lipid sufficed to divert phagosome maturation and likely contributes to mycobacterial survival in macrophages. Previous studies showed that activated macrophages promote maturation of mycobacterial phagosomes and eliminate mycobacteria through bactericidal effectors including nitric oxide generated by inducible nitric-oxide synthase. We show that deceleration of bead phagosome maturation by trehalose dimycolate was abolished in immune-activated wild type, but not in activated nitric-oxide synthase-deficient macrophages, nor when hydroxyl groups of trehalose dimycolate were chemically modified by reactive nitrogen intermediates. Thus, specific host defence

Received 29 November, 2007; revised 14 March, 2008; accepted 16 March, 2008. *For correspondence. E-mail ulrich.schaible@ Ishtm. ac.uk; Tel. (+44) 207958 8209; Fax (+44) $2079272807 .{ }^{\dagger}$ Present address: The Rockefeller University, Laboratory of Developmental Neurogenetics, New York, 10065, USA. effectors of activated macrophages directly target a specific virulence function of mycobacteria.

\section{Introduction}

Mycobacterium tuberculosis is the causative agent of tuberculosis, which is a major bacterial infection in humans worldwide killing two million people annually (Kaufmann and McMichael, 2005). M. tuberculosis and other pathogenic mycobacteria, such as Mycobacterium bovis and Mycobacterium avium, are facultative intracellular pathogens, which survive, proliferate and persist within macrophages $(M \Phi)$. While this intracellular survival strategy is not only essential for mycobacterial pathogenesis, it also represents a bacterial trait potentially accessible for novel therapeutic measures. $М \Phi$ are key players of innate immunity because of their ability to engulf and eliminate bacteria. Conventionally, $M \Phi$ enwrap bacteria into phagosomes, which mature to acidic and hydrolytic phagolysosomes. This process leads to killing and degradation of bacteria by an array of microbicidal and hydrolytic compounds. Persistence of pathogenic mycobacteria relies on their capability to exploit vesicular trafficking and to modify host cellular functions. Live mycobacteria arrest phagosomes at an early maturation stage. They retain early endosomal markers, such as the small GTPase Rab5, transferrin (Tf) and its receptor (TfR), and do not acidify below $\mathrm{pH} 6.2$ because of exclusion of the vacuolar proton-pumping ATPase (v-ATPase) (Russell, 2005). Although progression to later stages is inhibited, mycobacterial phagosomes remain accessible to early endosomes of the sorting/recycling pathway (Russell et al., 1996). This imports Tf-bound iron into the mycobacterial phagosome, which is an essential but limited growth factor for intracellular mycobacteria (Clemens and Horwitz, 1996; Sturgill-Koszycki et al., 1996; Schaible and Kaufmann, 2004; Wagner et al., 2005).

To address the question of how mycobacteria arrest phagosome maturation, several modes have been suggested, including mycobacteria-derived ammonia, which could buffer the phagosomal content (Gordon et al., 1980). A mycobacterial lipid phosphatase (SapM) was proposed as an inhibitor of vesicle fusion through cleavage of phosphatidylinositol 3-phosphate $[\mathrm{PI}(3) \mathrm{P}]$ in the phagosomal membrane (Vergne et al., 2005). As PI(3)P interacts with Rab5 and its effector, early endosomal 
antigen 1 (EEA1), to promote endosome maturation, a failure to recruit or maintain $\mathrm{PI}(3) \mathrm{P}$ on the phagosomal membrane could arrest a phagosome at an early stage. Other studies have demonstrated $\mathrm{PI}(3) \mathrm{P}$ retention on mycobacterial phagosomes (Kelley and Schorey, 2004; Purdy et al., 2005). Mycobacterial lipids have also been implicated in interfering with phagosome maturation as fostered by the observation that delipidated mycobacteria lost their capability to arrest phagosome maturation (Indrigo et al., 2002). The mycobacterial cell wall contains an array of genus- or even species-specific glycolipids, such as phosphatidylinositol-mannosides (PIM) and lipoarabinomannans (LAM). Functions of these lipids in phagosome maturation arrest have been attributed to their physicochemical similarity to $\mathrm{PI}(3) \mathrm{P}$, i.e. the phosphatidyl anchor, which could compete for EEA1 binding to the phagosomal membrane lipids (Fratti et al., 2003; Vergne et al., 2003; 2004). A different scenario suggests that ligation of mannose receptors through mannosylated glycolipids on the mycobacterial surface, such as LAM and PIM, delivers mycobacteria to early endosomal compartments (Kang et al., 2005). Previously, we found that mycobacteria, isolated from MФ $24 \mathrm{~h}$ post infection (p.i.) contained almost 50\% more trehalose dimycolate (cord factor; TDM) when compared with those purified at $2 \mathrm{~h}$ p.i. This indicates that mycobacteria increase TDM production in response to the intracellular environment (Fischer et al., 2001). Together with the seminal report by Indrigo et al. (2003) that phagosomes containing TDM-coated beads do not fuse with lysotracker-positive compartments, this suggests a virulence function of TDM for intracellular survival of mycobacteria.

Employing different assays, we demonstrate here that, in comparison with normal bead phagosomes, maturation of phagosomes containing beads coated with TDM is decelerated and retarded at an early, non-acidified and hydrolytically restricted stage similar to fully established mycobacterial phagosomes. More importantly, this virulence function of TDM is fully abrogated in interferon gamma (IFN- $\gamma$ )/LPS-activated $M \Phi$ involving nitric-oxide synthase (NOS-2) and reactive nitrogen intermediates (RNI). This reveals direct inactivation of a single mycobacterial molecule with an essential virulence function by unique macrophage-specific microbicidal effectors.

\section{Results}

Trehalose dimycolate inhibits bead phagosome maturation

In order to test whether TDM influences phagosome maturation, TDM and its precursor trehalose monomycolate (TMM) were purified from M. bovis Bacille Calmette Guerin (BCG) as described (Fischer et al., 2001; 2002) and equal amounts were absorbed onto BSA-coated magnetic beads (hereafter called TMM or TDM beads). Coating of equal amounts of lipids was verified by re-extraction and high performance thin layer chromatography (HPTLC) analysis of lipids from coated beads (Fig. S1C). Coated beads were bound to $M \Phi$ using a magnet, washed and incubated at $37^{\circ} \mathrm{C}$ for the times indicated to follow intracellular fate of beads by fluorescence and confocal laser scanning microscopy (LSM), by intraphagosomal pH measurement and immunochemical Western blot analyses of purified bead phagosomes. The intracellular localization of BSA beads coated with or without lipids was studied in primary bone marrow-derived $\mathrm{M} \Phi$ or RAW-B $M \Phi$ expressing the $\mathrm{PI}(3) \mathrm{P}$-binding domain PX fused to GFP (PX-GFP) (Ellson et al., 2001). Upon phagocytosis, trafficking of bead phagosomes was followed using tracers and antibodies to marker proteins in order to differentiate between distinct endosomal maturation stages (Schaible and Kaufmann, 2002). In BSA bead phagosomes, early endosomal proteins $\mathrm{Tf}$ and TfR were present between 5 and 10 min after phagocytosis, but absent at later time points (20, $30 \mathrm{~min})$ when late endosome lysosome markers, lysosomeassociated membrane protein 1 (LAMP-1) and dextran Texas Red (dexTR), a fluid phase tracer pre-accumulated in lysosomes, were delivered to these phagosomes (data not shown). The kinetics of BSA bead phagosome maturation reflects the rapid and conventional process as described earlier for bead phagosomes (Sturgill-Koszycki et al., 1996; Scott et al., 2003). Similar to BSA bead phagosomes, those containing TMM-coated beads carried Tf and TfR $10 \mathrm{~min}$, and LAMP-1 and dexTR $30 \mathrm{~min}$ after phagocytosis (Fig. 1A and B; Fig. S1 A and B). In contrast, most TDM bead phagosomes retained Tf and TfR for more than 30 min after phagocytosis and did not accumulate dexTR (Fig. 1A and B; Fig. S1 A, B and D). Some LAMP-1 was recruited to TDM bead phagosomes, which is in line with reports that LAMP-1 is also present in mycobacterial phagosomes in primary $\mathrm{M} \Phi$ of both murine and human origins (Clemens and Horwitz, 1995; Sturgill-Koszycki et al., 1996). $\mathrm{PI}(3) \mathrm{P}$, which transiently accumulates in early phagosome membranes, was studied in RAW MФ stably expressing the PI(3)P-binding PX domain of the NADPH oxidase fused to GFP (Ellson et al., 2001). BSA bead phagosomes (Fig. S2A and B) as well as TMM bead phagosomes (Fig. 1C and D) were positive for $\mathrm{PI}(3) \mathrm{P} 10 \mathrm{~min}$ but negative $30 \mathrm{~min}$ after phagocytosis, demonstrating the transient nature of its recruitment during phagosome maturation. Of note, $\mathrm{PI}(3) \mathrm{P}$-positive bead phagosomes did not contain LAMP-1, while the absence of PI(3)P on bead phagosomes 30 min after uptake was accompanied by the presence of LAMP-1. Thus, the RAW M $\Phi$ cell line differs from primary $M \Phi$ in the kinetics of LAMP-1 recruitment to maturing phagosomes, in line with observations by others (Gutierrez et al., 2004). In contrast to BSA and 
Fig. 1. A. TDM excludes dexTR from bead phagosomes. Bone marrow-derived $M \Phi$ were incubated with TMM or TDM beads for 10 or 30 min. Lysosomes/late endosomes were labelled by a $2 \mathrm{~h}$ dexTR pulse followed by a $2 \mathrm{~h}$ chase. Cells were fixed and stained for TfR, which was visualized using a CY2-labelled secondary antibody. Arrowheads indicate bead phagosomes.

B. Quantification of dexTR and TfR staining in 100 bead phagosomes showing means \pm standard deviation of three independent experiments. C. TDM beads maintain PI(3)P-positive phagosomes. RAW MФ expressing PX-GFP were incubated with TMM or TDM beads or Texas Red-labelled M. avium (lower row, arrowheads indicate mycobacteria) for $30 \mathrm{~min}$. Cells were fixed and immunostained for LAMP-1 (only bead-containing cells).

D and E. Quantification of PX-GFP and LAMP-1 staining in 100 bead (D) and/or 50 M. avium phagosomes (E), showing means \pm standard deviation of three independent experiments. Inserts: magnified images of representative bead phagosomes. Bar: $10 \mu \mathrm{m}$.

F. TDM is not released from beads in detectable amounts. MФ $\left(5 \times 10^{6}\right)$ were incubated with TDM beads $(5: 1)$ for 1 and $2 \mathrm{~h}$. Cells were washed and disrupted by freeze thaw. Beads were purified from the cellular fractions by magnet. Both fractions were extracted in chloroform/methanol (2:1) and separated by HPTLC in chloroform/methanol/acetone/acetic acid (90:10:6:1) and visualized by sulfuric acid spray and charring at $200^{\circ} \mathrm{C}$. Lanes: purified TDM $15 \mu \mathrm{g}(1), 7.5 \mu \mathrm{g}(2), 1 \mu \mathrm{g}(3), 0.2 \mu \mathrm{g}$ (4), extracts from purified TDM beads (5) and cell fraction after $1 \mathrm{~h}(6)$, and $2 \mathrm{~h}(7,8)$, respectively, and TDM beads before incubation with cells (9). Equivalents of $5 \times 10^{6}$ beads were loaded in lanes $5-8$, and of $5 \times 10^{5}$ in lane 9 . The position of TDM is indicated by an arrow.

TMM bead phagosomes, TDM bead phagosomes retained $\mathrm{PI}(3) \mathrm{P}$ and excluded LAMP-1 for more than 30 min after phagocytosis (Fig. $1 \mathrm{C}$ and D). PI(3)P synthesis at the mycobacterial phagosome membrane has been reported before (Chua and Deretic, 2004; Kelley and Schorey, 2004; Purdy et al., 2005). By using live $M$. avium, $M$. tuberculosis and $M$. bovis BCG, we confirmed that $\mathrm{PI}(3) \mathrm{P}$ persisted on phagosomes containing live mycobacteria for $30 \mathrm{~min}$ to $1 \mathrm{~h}$ after uptake, indicating that these compartments are at the early/intermediate maturation stage (Fig. 1C, lower panel, E, Fig. S2C and D). However, already $4 \mathrm{~h}$ p.i., more than $60 \%$ of the $M$. tuberculosis and $M$. bovis BCG phagosomes became negative for PX-GFP. Twenty-four hours post infection, all mycobacterial phagosomes were free of $\mathrm{PI}(3) \mathrm{P}$ (Fig. S2C and D). This indicates that, although both mycobacterial species maintained $\mathrm{PI}(3) \mathrm{P}$ synthesis on phagosomes for a longer period when compared with BSA bead phagosomes, this is still a transient event in mycobacterium's phagosomal fate.

It should be noted that the inhibitory effect of TDM was most striking at $30 \mathrm{~min}$ after uptake and became less apparent at later time points, i.e. at 1 and $2 \mathrm{~h}$ (Fig. S1D). This discrepancy between TDM bead and mycobacterial phagosomes is likely due to decreasing TDM amounts on beads, but continuous de novo synthesis of TDM by life mycobacteria. In order to analyse whether TDM is released from beads within phagosomes, we separated TDM beads from cellular membranes 1 and $2 \mathrm{~h}$ after uptake by $M \Phi$, and lipids were extracted from both fractions. As shown by HPTLC, 1 and $2 \mathrm{~h}$ after uptake, TDM was found in similar amounts in the bead fractions, but was not detectable in the cellular membrane fraction, suggesting that only limited amounts not detectable by this method were released during a $2 \mathrm{~h}$ incubation period (Fig. 1F).

Close contact between phagosomal membrane and particle has been described for immature, early mycobacterial phagosomes as well as for phagosomes containing beads with a hydrophobic surface (de Chastellier and
Thilo, 1997; Schaible et al., 1998). At 10 min after uptake, both BSA and TDM beads were found in tight phagosomes (data not shown). TDM beads maintained close contact to the phagosomal membrane 30 min after uptake when BSA bead phagosomes had already become spacious, a hallmark of late phagosomes (Fig. 2C; upper two panels) (de Chastellier and Thilo, 2006). This suggests that TDM-mediated retardation of phagosome maturation is characterized by extended maintenance of the tight association between phagosomal membrane and bead surface.

To further evaluate the inhibitory effects of TDM on phagosome maturation, bead phagosomes were purified from МФ 30 min after uptake. Equal amounts of phagosome proteins were analysed by Western blot using antibodies to compartment-specific marker proteins. TMM bead phagosomes contained in general more mature Cathepsin D than TDM bead phagosomes (Fig. S3 A and B). There was less Procathepsin D, v-ATPase and LAMP-1 but more Rab5 in TDM bead compared with TMM bead phagosomes (Fig. S3A and B). The immature character of purified TDM compared with TMM bead phagosomes was also manifested by reduced activities of the lysosomal enzymes, $\beta-\mathrm{N}$-hexosaminidase and $\beta$-galactosidase (Fig. S3C). In summary, TDM bead phagosomes were arrested at an early endosome stage and acquired neither v-ATPase nor lysosomal enzyme activity in appreciable amounts.

\section{Trehalose dimycolate bead phagosomes fail to acidify}

While phagosomes containing non-pathogenic particles rapidly acidify from an almost neutral $\mathrm{pH}$ to a $\mathrm{pH}$ below 5.0 , mycobacterial phagosomes maintain a $\mathrm{pH}$ between 6.2 and 6.4 by exclusion of v-ATPase (Sturgill-Koszycki et al., 1994; Yates et al., 2005). Both heat and irradiation killing can alter the cell wall lipid content and intracellular trafficking of mycobacteria (Oh and Straubinger, 1996; Kang et al., 2005), but some reports show that killed mycobacteria inhibit phagosome maturation to some extent and 


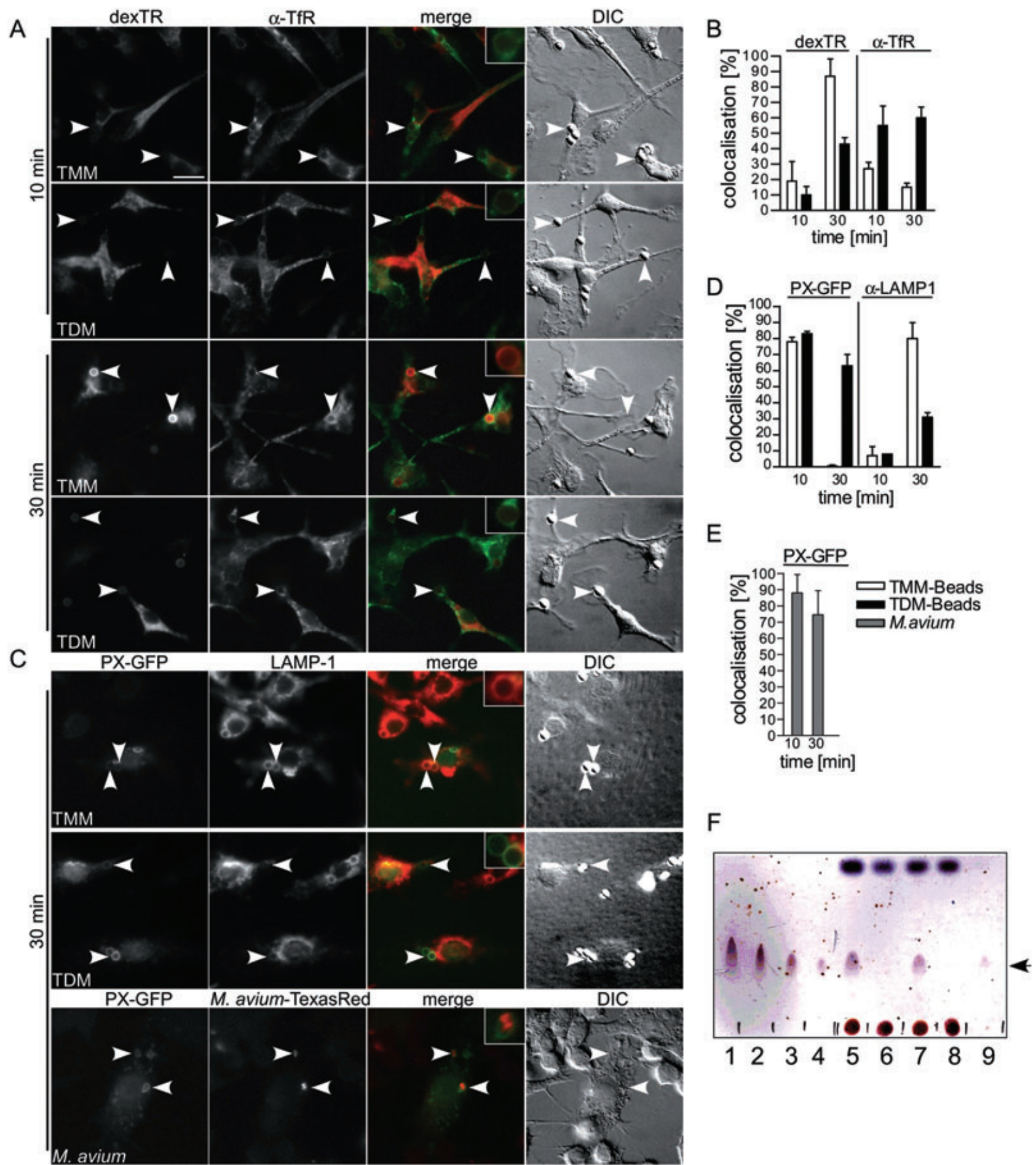

maintain a pH of around 5.5 (Russell, 2005). LysoSensor Yellow/Blue, an acidotropic $\mathrm{pH}$ indicator with $\mathrm{pH}$ dependent dual excitation and dual emission spectral peaks, was employed to follow acidification of individual bead phagosomes. Fluorescence in BSA and TMM bead phagosomes changed from blue (shown in red) to green between 8 and 18 min after uptake (Fig. 2A; Fig. S3D) with a drop of $\mathrm{Em}_{445 \mathrm{~nm}} / \mathrm{Em}_{560 \mathrm{~nm}}$ ratio from 1.4 to 0.8 (Fig. 2B), indicating a rapid decrease of phagosomal $\mathrm{pH}$. In contrast, fluorescence remained unchanged in TDM bead phagosomes as reflected by an unaltered $\mathrm{Em}_{445 \mathrm{~nm}} / \mathrm{Em}_{560 \mathrm{~nm}}$ ratio of 1.4 at the 18 min time point (Fig. 2A and B). Thus, presence of TDM prevented acidification because of exclusion of the v-ATPase from these phagosomes (Fig. S3 A and B) 

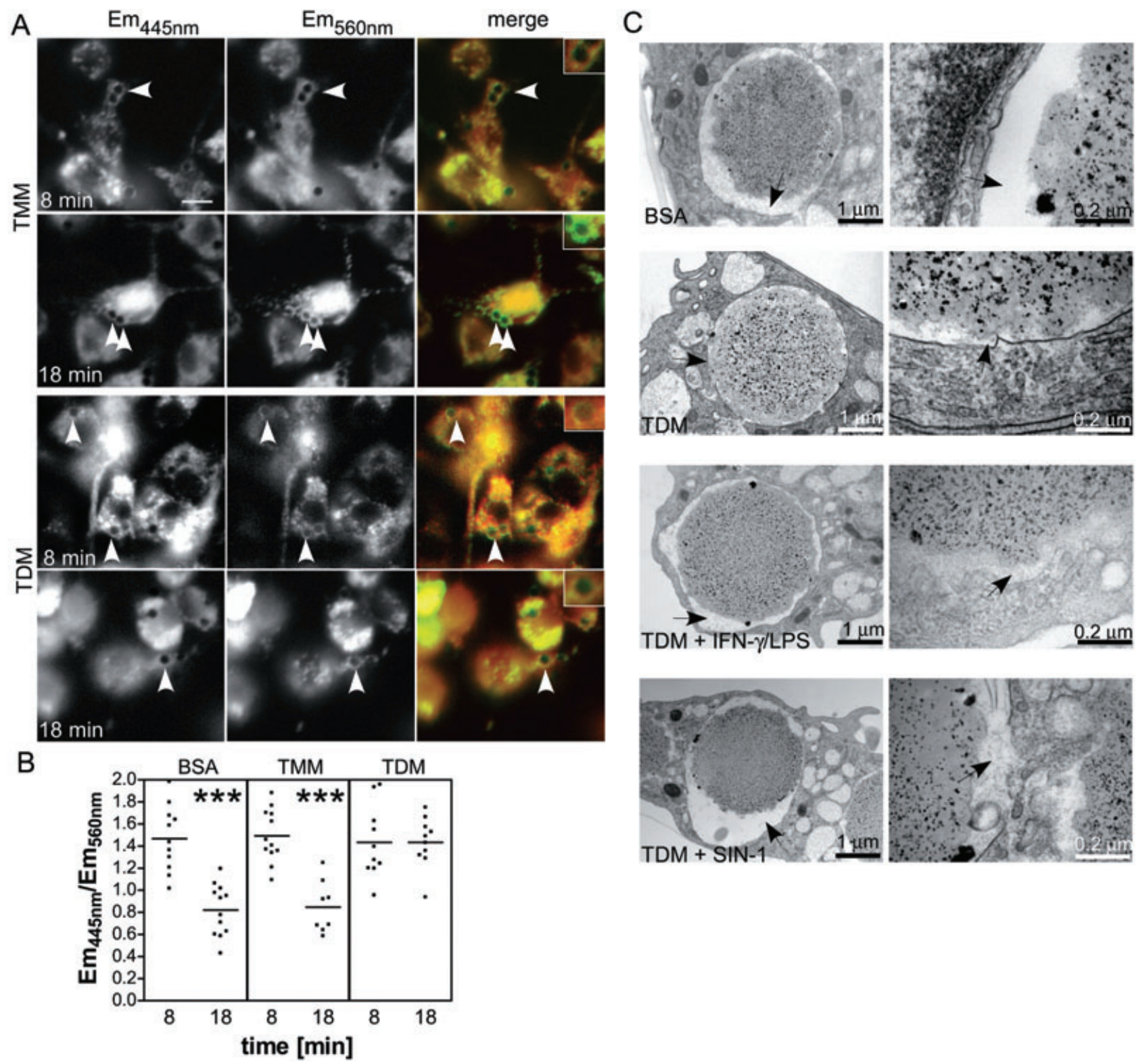

Fig. 2. A. TDM bead phagosomes do not acidify. M $\Phi$ were incubated with TDM- or TMM beads in the presence of $15 \mu M$ LysoSensor Yellow/Blue for 8 or $18 \mathrm{~min}$ and chased for $8 \mathrm{~min}$. Cells were analysed by fluorescence microscopy using dual excitation and emission filters. Fluorescent micrographs were recorded at $\mathrm{Ex}_{360 \mathrm{~nm}} / \mathrm{Em}_{445 \mathrm{~nm}}$ (blue) and $\mathrm{Ex}_{380 \mathrm{~nm}} / \mathrm{Em}_{560 \mathrm{~nm}}$ (green). Acidic compartments appear in green and neutral ones in red. Arrowheads indicate bead phagosomes. Bar: $10 \mu \mathrm{m}$.

B. Quantification of bead phagosome acidification. Plots show blue/green ratios of 10 individual phagosomes including means

(statistics: unpaired Student's t-test). For representative pictures of BSA bead phagosomes see Fig. S3D.

C. TDM-coated beads maintain close contact to the phagosomal membrane. Transmission electron micrographs of bead phagosomes 30 min after phagocytosis. Upper two panels: phagosomes containing beads either coated with BSA or TDM. Third panel: TDM bead phagosomes in M $\Phi$ activated with IFN- $\gamma /$ LPS for $24 \mathrm{~h}$. Lower panel: Phagosomes with SIN-1-treated TDM beads. Arrows indicate the contact area between bead and phagosomal membrane.

(Sturgill-Koszycki et al., 1994). As TDM alone is sufficient to arrest bead phagosome maturation at a stage which resembles phagosomes containing live mycobacteria, we consider TDM to be a key factor in the inhibition of phagosome maturation by mycobacteria.
Macrophage activation interferes with inhibition of phagosome maturation by TDM

Resting $M \Phi$ shelter mycobacteria from humoral defence mechanisms and promote bacterial growth. However, 
IFN- $\gamma$ activates microbicidal $M \Phi$ functions essential for protective immunity against tuberculosis. Autocrine tumour necrosis factor alpha (TNF- $\alpha$ ) production further exacerbates these effector functions (Martin et al., 1994) As macrophage activation also promotes maturation of mycobacterial phagosomes (Schaible et al., 1998), we followed trafficking of TDM beads in activated MФ. Cells were activated by either IFN- $\gamma$ or LPS (to stimulate TNF- $\alpha$ production), and subsequently fed with TDM-coated beads. These were transiently present in early compartments as seen with resting $М \Phi$. However, the number of phagosomes which matured further towards a late endosomal/lysosomal stage within $30 \mathrm{~min}$ was increased in activated compared with resting $М \Phi$ (Fig. 3; Fig. S4). More importantly, in $\mathrm{M} \Phi$ activated by a combination of IFN- $\gamma$ and LPS, inhibition of phagosome maturation by TDM was fully abolished. Under these conditions, TDM bead phagosomes acquired LAMP-1, mature Cathepsin $\mathrm{D}$ as well as v-ATPase, and they fused with dexTR-loaded lysosomes. Yet, they became negative for $\mathrm{TfR}$, Tf and $\mathrm{PI}(3) \mathrm{P}$ (Fig. 3B, D and E; Fig. S4 A-C). Acidification of TDM bead phagosomes in M $\Phi$ activated with either LPSor IFN- $\gamma$ followed similar kinetics as BSA and TMM bead phagosomes (Fig. 3A and C versus Fig. 2B). However, TDM phagosomes in IFN- $\gamma /$ LPS-activated M $\Phi$ had already acidified 8 min after engulfment, which occurred significantly faster than acidification of BSA bead phagosomes in resting $\mathrm{M} \Phi$ (Fig. $3 \mathrm{C}$ versus Fig. $2 \mathrm{~B}$ ). In addition, 10 min after uptake, TDM bead phagosomes had already acquired late endosomal/lysosomal characteristics in IFN$\gamma /$ LPS-activated but not resting $M \Phi$ or those activated by either LPS or IFN- $\gamma$ (Fig. 3D and E). Therefore, trafficking of TDM beads in activated $\mathrm{M} \Phi$ was strikingly different from what has been observed in resting $M \Phi$, i.e. targeting them into phagolysosomes.

\section{Reactive nitrogen intermediates inactivate TDM}

We hypothesized that microbicidal effectors of activated MФ may target TDM. Among other genes, IFN- $\gamma$ induces and enhances expression of NOS-2, which catalyses production of toxic RNI from arginine. We observed recruitment of NOS-2 to the membrane of TDM bead phagosomes upon stimulation of M $\Phi$ with IFN- $\gamma /$ LPS as shown by Western blot of purified bead phagosomes (Fig. 4A). To test our hypothesis that RNI may affect TDM, we employed M $\Phi$ from mice deficient for NOS-2 in comparison with those from their wild-type littermates to follow maturation of TDM bead phagosomes upon activation. In resting $\mathrm{M} \Phi$ of both wild-type and NOS-2-deficient mice 30 min after uptake, TDM beads resided predominantly in non-acidified phagosomes (Fig. 4D) positive for Tf and TfR (Fig. S5A-D), when TMM and BSA bead phagosomes were already found in phagolysosomes (data not shown). In contrast, activation of wild-type $\mathrm{M} \Phi$ forced TDM bead phagosome maturation towards phagolysosomes. However, activation of NOS-2-deficient M $\Phi$ by IFN- $\gamma$, LPS, or both, failed to promote maturation (Fig. 4D; Fig. S5 A-D). Further confirmation that NO counteracts TDM's virulence function was revealed by experiments showing that specific NOS-2 inhibition by $\mathrm{N}$ (G)-nitro-L-arginine methyl ester (L-NAME) prevented inactivation of TDM in activated MФ (Fig. S6A and B). In summary, these experiments revealed that in activated $M \Phi$, newly phagosomal NOS-2 affects TDM's virulence function.

In order to investigate direct effects of NOS-2 products, RNI, on TDM, we treated TDM-coated beads with chemically defined donors of nitric oxide (NO) and superoxide, or NO alone, 3-morpholinosydnomine (SIN-1) and sodium nitro-prusside (SNP) respectively. In contrast to mocktreated TDM beads, those treated with SIN-1 or SNP quickly trafficked to phagolysosomes as revealed by loss of PI(3)P (Fig. 4B and C) and acidification (Fig. 4E; Fig. S7; shown for SIN-1). Moreover, the tight association between TDM beads and the phagosomal membrane as observed by electron microscopy, was lost in activated $\mathrm{M} \Phi$ as well as upon treatment with SIN-1 (Fig. 2C, lower two panels). Hence, RNI can fully abolish TDM-mediated inhibition of phagosome maturation suggesting chemical modification of TDM.

In order to characterize the modifications catalysed by RNI, mock or SIN-1-treated TDM, samples were analysed by HPTLC and nuclear magnetic resonance (NMR). HPTLC of both treated and untreated lipids revealed comparable migration patterns (data not shown). NMR spectroscopy yielded spectra of homogeneous preparations. The ${ }^{1} \mathrm{H}-\mathrm{NMR}$ spectra showed isolated signals of olefinic protons around 6-7.5 p.p.m. (data not shown), signals of the sugar moieties in the range of 2.5-6 p.p.m. and lipid signals below 2 p.p.m. The ${ }^{1} \mathrm{H}$-signals of the $\alpha$ and $\beta$ methine carbons of the mycolic acids are expected around 2.5 and 3.5 p.p.m. respectively (Fig. 5A). 1D and 2D ${ }^{1} \mathrm{H}-13 \mathrm{C}$ correlation spectra were recorded to resolve signal overlap and to allow assignments of protons based on the chemical shifts of the directly connected carbons (Fig. 5). Regarding the spectra of TDM treated with SIN-1, NMR spectroscopy revealed spectral changes in the range of the sugar signals at 4.3 and 4.35 p.p.m., as well as around 3.55 and 3.8 p.p.m., when compared with spectra of mocktreated lipids [affected signals are indicated by arrows $(\mathrm{SIN}-1)$ or stars (mock)], in line with an expected modification by $\mathrm{SIN}-1$ at the $\mathrm{OH}$ groups (Fig. 5A and B). The spectral changes in the region of around 3.55 p.p.m. are possibly due to an interaction with the mycolic acid hydroxyl $(\mathrm{OH})$ group (arrow). The reaction with the sugar moiety as indicated by the change of signals above 
Fig. 3. $M \Phi$ activation interferes with TDM-mediated inhibition of phagosome maturation.

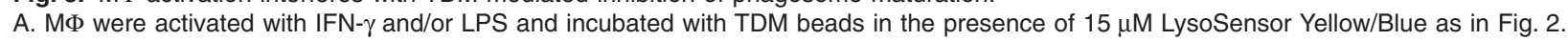
B. RAW M $\Phi$ expressing PX-GFP were activated with IFN- $\gamma$ and/or LPS and incubated with TDM beads for 30 min. Cells were fixed and stained for LAMP-1.

C. Quantification of phagosomal acidification showing blue/green ratios of 10-13 individual phagosomes, including means of one representative out of three independent experiments (statistics: unpaired Student's $t$-test).

D. M $\Phi$ were activated with IFN- $\gamma$ and/or LPS and incubated with TDM beads for 10 and $30 \mathrm{~min}$. Cells were labelled for early endosomes or late endosomes/lysosomes with Tf-CY3 or dexTR. After fixation, cells were stained for TfR or LAMP-1 and analysed by fluorescence microscopy. The graph shows the quantification of phagosomal colocalization with the respective markers as means \pm standard deviation of three independent experiments. See Fig. S4A and B for representative pictures.

E. Quantification of PI(3)P (PX-GFP) and LAMP-1 staining of 100 bead phagosomes in activated versus non-activated RAW M $\Phi$ incubated with beads for 10 and $30 \mathrm{~min}$, showing means \pm standard deviation of three independent experiments. Inserts: magnified images of one representative bead phagosome. Bars: $10 \mu \mathrm{m}$.

4.0 p.p.m., i.e. at 5.4 and 5.7 p.p.m., was quantitative, as signals of the mock-treated form vanished (see signals indicated by arrows) and new signals occurred in the treated one (Fig. 5A and B; signals missing in either spectrum are indicated by empty circles). As shown in Fig. 5B, which represents the magnified spectrum between 3.1 and 4.6 p.p.m. of an independent preparation, there were additional changes in the sugar signals inflicted by the NO donor. In this preparation, the signals at 4.3 and 3.65 p.p.m. completely disappeared upon SIN-1 treatment, whereas the corresponding signals in the spectrum depicted in Fig. 5A are only diminished. On the whole, the number of signals remained unaffected, and there were no chemical shift changes observed in the region of the olefinic signals between 6 and 7.5 p.p.m. (data not shown), suggesting that the olefinic groups within the lipid chain were unaltered. Hence, intact hydroxyl groups are essential moieties of TDM in inhibition of phagosome maturation and are specifically targeted by $\mathrm{RNI}$ in activated $\mathrm{M} \Phi$.

\section{Discussion}

Increased production of TDM by intracellular mycobacteria prompted us to analyse TDM function in phagosome maturation (Fischer et al., 2001). Our studies revealed that TDM, when coupled onto beads, slowed down phagosome maturation and prolonged the retention period in an early, non-acidified and hydrolytically restricted stage characteristic for mycobacterial phagosomes. However, this virulence function of TDM was fully abolished upon activation of M $\Phi$ by IFN- $\gamma /$ LPS in a NOS-2-dependent manner. We show that RNI directly inactivated TDM's virulence function by altering its hydroxyl groups.

Trehalose dimycolate has been suggested to be essential for mycobacterial cell wall rigidity and stability and was always a prime candidate for virulence functions (Barry et al., 1998): (i) of all mycobacterial lipids, it is one of the strongest inducers of inflammatory responses (Geisel et al., 2005); (ii) cyclopropyl modification of $\alpha$-mycolic acid in TDM was only found in pathogenic mycobacteria, and a strain lacking these modified mycolic acids showed reduced virulence (Yuan et al., 1998; Rao et al., 2006); (iii) TDM may scavenge reactive oxygen intermediates (ROI) and RNI, thereby protecting mycobacteria from MФ effectors (Barry et al., 1998); (iv) delipidated mycobacteria failed to arrest phagosomal maturation, but reconstitution with TDM restored this ability (Indrigo et al., 2002); (v) an attenuated $M$. tuberculosis deletion mutant for antigen 85 A (fbpA), a mycoloyl transferase involved in TDM synthesis, was unable to block phagosome maturation (Copenhaver et al., 2004) and (vi) finally, TDM coupled to latex beads was found to inhibit fusion of phagosomes with lysotracker-labelled lysosomal compartments (Indrigo et al., 2003).

Using four different assays, we demonstrate here that TDM single-handedly has the same impact on phagosome maturation as live mycobacteria, at least within the first $30 \mathrm{~min}$ after phagocytosis. This is astonishing because beads can bind and release only a limited amount of lipid, whereas live mycobacteria can continuously renew TDM and other glycolipids and therefore permanently impair host cell functions (Beatty et al., 2000; Fischer et al., 2001). A genetic approach to unfold TDM's function on phagosome maturation is unfortunately not feasible as TDM is essential for integrity of the mycobacterial cell wall. Deletion mutants of TDM biosynthesis genes are already impaired for growth in broth culture (Copenhaver et al., 2004). The single-lipid-on-bead model is therefore a simplistic yet valid approach to study a single virulenceassociated lipid of a complex pathogen (Fratti et al., 2003).

The question remains how TDM interferes with phagosome maturation and a number of scenarios can be envisaged. We observed that TDM-mediated inhibition of phagosome maturation is characterized by maintenance of a tight association between phagosomal membrane and bead surface as described for mycobacterial phagosomes (Schaible et al., 1998). This may indicate that an intimate interaction during phagocytosis between TDM and either cell-surface membrane lipids or receptors is maintained after internalization. Engaging surface receptors may specifically target intracellular compartments as recently suggested for manLAM binding to the mannose receptor, but specific receptors for TDM have not yet been 


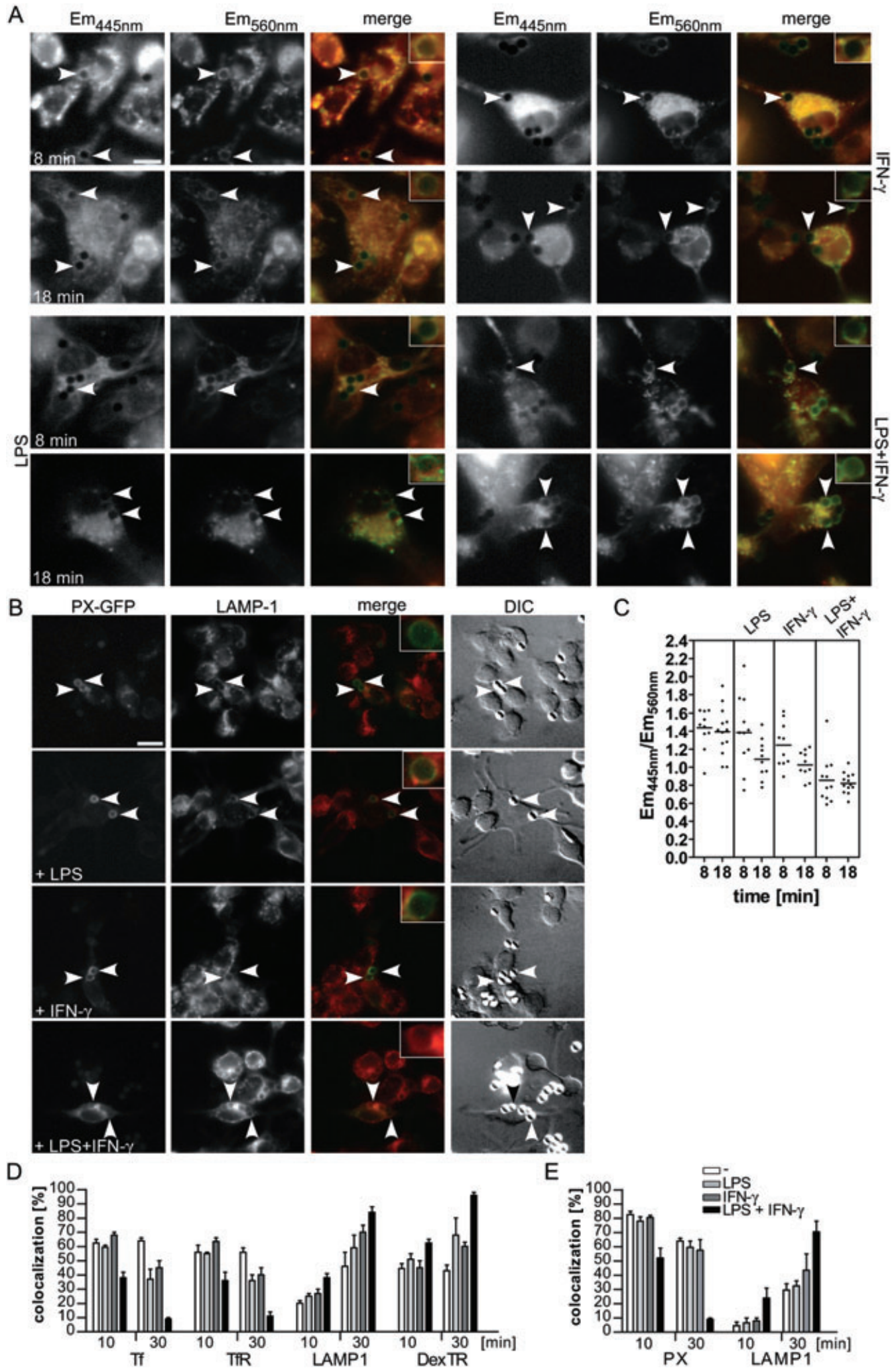


A $B$

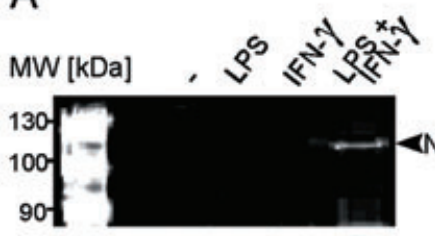

B
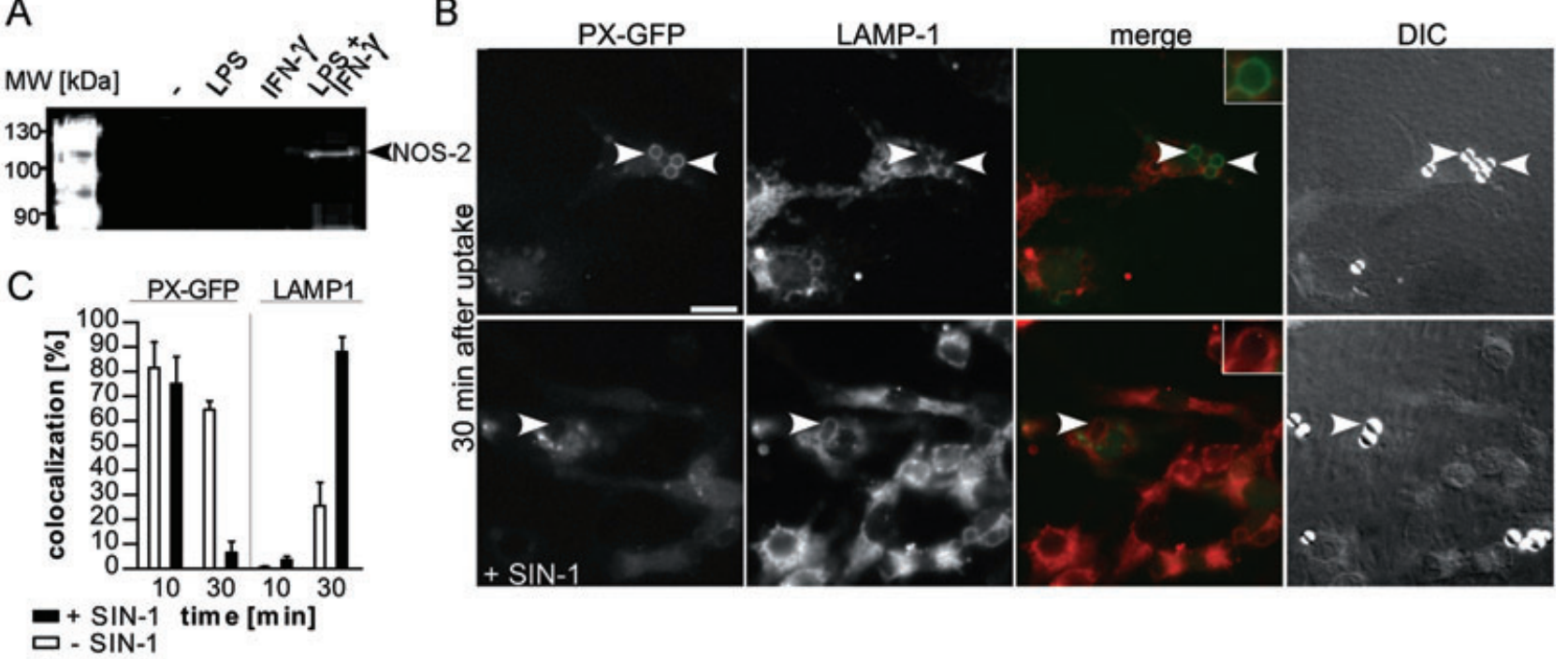

口 - SIN-1

D

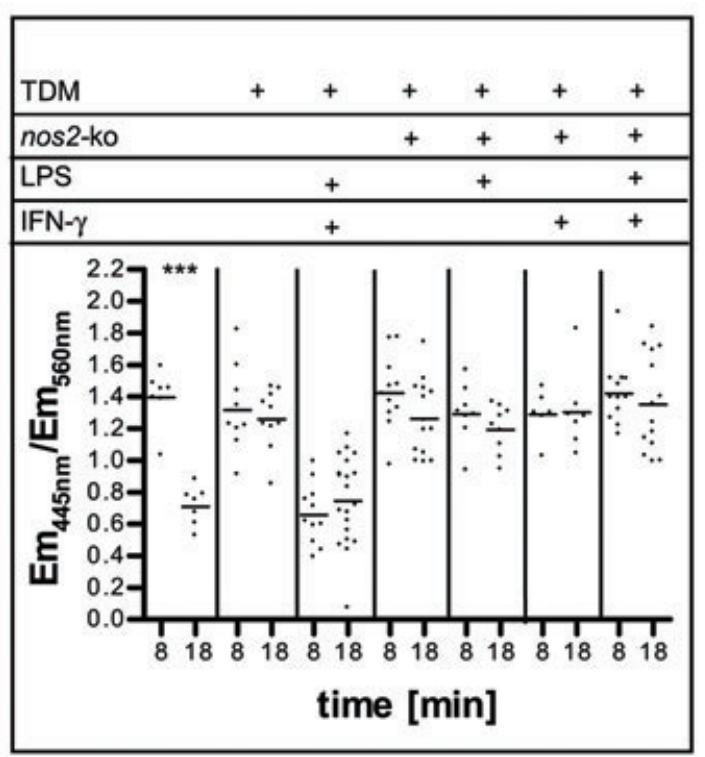

E

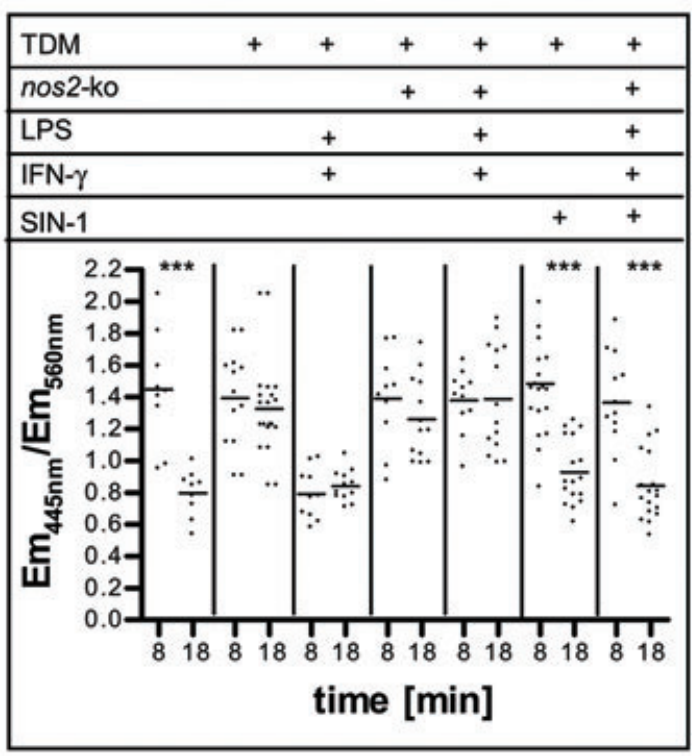

Fig. 4. A. NOS-2 is present on membranes of TDM bead phagosomes in IFN- $\gamma /$ LPS-activated M $\Phi$. Western blot of TDM bead phagosomes purified from cells activated with IFN- $\gamma$ and/or LPS and subsequently incubated for 30 min with TDM beads. The blot was developed using anti-NOS-2 antibodies. Molecular weight markers are shown on the left.

B. Nitric oxide abrogates TDM-mediated inhibition of phagosome maturation. RAW MФ expressing PX-GFP were incubated with BSA beads coated with mock- or SIN-1-treated TDM for $30 \mathrm{~min}$. Cells were fixed and stained for LAMP-1. Bar: $5 \mu \mathrm{m}$.

C. Quantification of PI(3)P (PX-GFP) and LAMP-1 staining of mock- or SIN-1-treated TDM bead phagosomes in PX-GFP expressing RAW $M \Phi$, showing means of 100 analysed phagosomes \pm standard deviation of three independent experiments.

D. NOS-2 interferes with TDM-mediated inhibition of phagosomal acidification. M $\Phi$ from either wild-type mice or nos-2-ko mice were activated with IFN- $\gamma$ and/or LPS and incubated with BSA beads (1st column) or TDM beads in the presence of $15 \mu \mathrm{M}$ LysoSensor Yellow/Blue as in Fig. 2. For quantification of phagosomal acidification, plots show blue/green ratios of 8-17 individual phagosomes, including means of one representative out of three independent experiments (statistics: unpaired Student's $t$-test; triple asterisks: $P<0.0001$ ). For representative

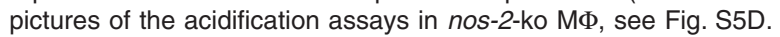

E. SIN-1 treatment annihilates TDM-mediated inhibition of phagosome acidification. BMMФ from either wide-type mice or nos-2-ko mice were activated as indicated and incubated with TDM beads or beads coated with SIN-1-treated TDM in the presence of $15 \mu \mathrm{M}$ LysoSensor Yellow/Blue as in Fig. 2. For quantification of acidification, plots show blue/green ratios of 9-18 individual phagosomes including means (statistics: unpaired Student's $t$-test; triple asterisks: $P<0.0001$ ). Shown is one representative out of three independent experiments. For representative pictures, see Fig. S7. 
A

\section{TDM SIN-1 treated}

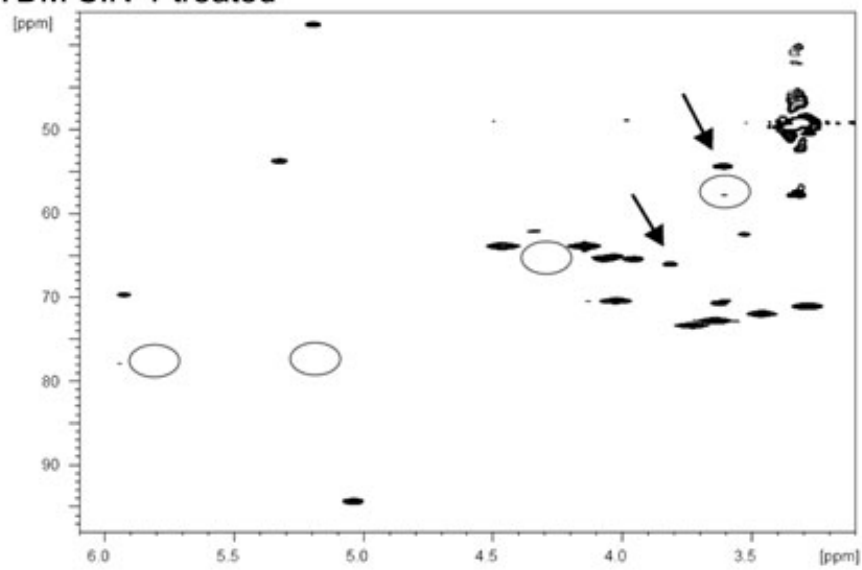

\section{TDM mock treated}

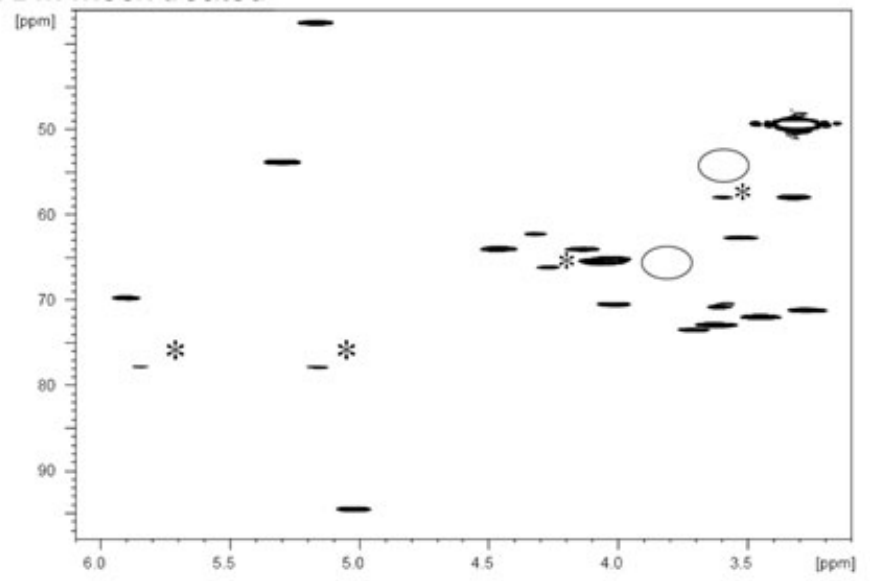

Fig. 5. NMR analysis reveals alteration of TDM's hydroxyl groups by nitric oxide. A. 2D 1H-13C correlation spectra of mockand SIN-1-treated TDM (bottom and top respectively). The arrows indicate signals that appear upon SIN-1 treatment compared with mock-treated TDM. Asterisks indicate spurious signals that are also present in the spectra of the mock-treated form, but at lower contour levels than shown. Circles indicate signals, which are absent in one but present in the other spectrum.

B. $1 \mathrm{H}-13 \mathrm{C}$ correlation spectra of mock- and SIN-1-treated TDM (right and left respectively) from two independent preparations. Shown are magnifications of the area covering the range between 3.1 and 4.7 p.p.m. and the corresponding 1D spectra. The arrows indicate signals that appear upon SIN-1 treatment compared with mock-treated TDM. Asterisks indicate spurious signals that are also present in the spectra of the

mock-treated form, but at lower contour levels than shown. Circles indicate signals which are absent in one but present in the other spectrum. Notably, in this preparation, signals at 4.3 p.p.m. and 3.65 p.p.m. disappear completely upon SIN-1 treatment, whereas the corresponding signals in Fig. 5A are only diminished.

\section{B TDM SIN-1 treated}

TDM mock treated
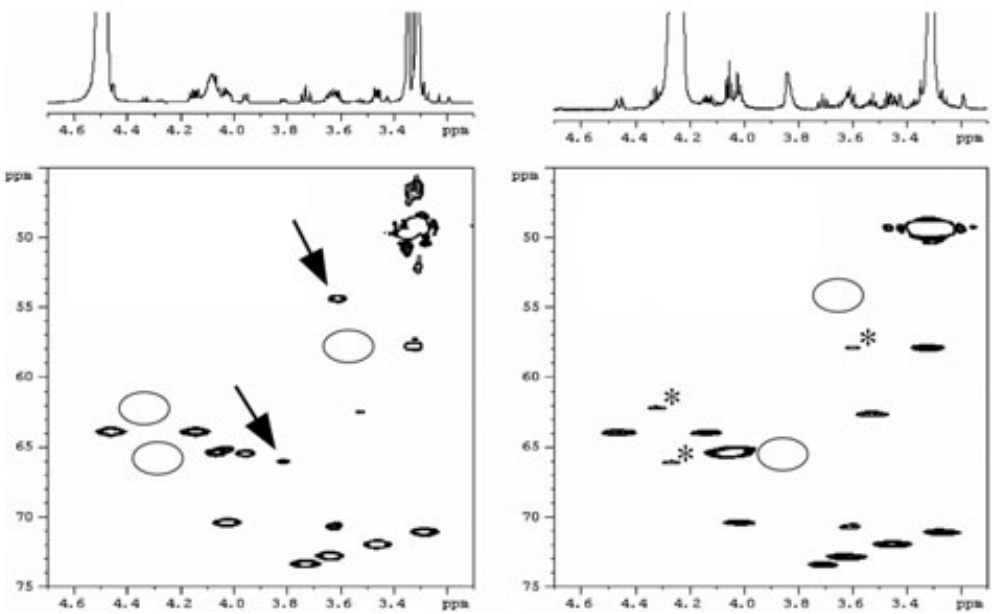

identified (Kang et al., 2005). Previous reports have also shown that a hydrophobic particle surface has close apposition to the phagosomal membrane. This interaction has been suggested by the authors as a possible mecha- nism for interference with phagosome maturation (de Chastellier and Thilo, 1997; 2006).

We found that TDM is not released from the beads in detectable amounts during a $2 \mathrm{~h}$ observation time, sug- 


\section{S. Axelrod et al.}

gesting that interference with phagosome maturation does not require intercalation into the phagosomal membrane, or at least not of vast amounts of TDM. In contrast, current models on TDM's effect on host membrane functions require non-bound molecules. Various non-specific effects resulting from lipid-lipid interactions have been described for TDM. A number of studies addressed interactions of TDM with model membranes. It was observed that TDM inhibits calcium-driven homotypic fusion of phospholipid vesicles, suggesting that integration of TDM's two long hydrophobic fatty acid chains (C50-70) into phospholipid bilayers may interfere with membrane integrity and stability, leading to acyl chain disorder, alteration of membrane curvature and disruption of lipid microdomains (Spargo et al., 1991; Crowe et al., 1994). Yet, TDM did not cause leakage of liposomes, but rather rigidified liposome membranes (Sut et al., 1990). The failure of TMM to influence phagosome maturation suggests that one mycolic acid chain is insufficient to interfere with vesicle fusion. Intercalation of TDM into membranes is promoted by phosphatidyl inositol (PI) within these membranes (Almog and Mannella, 1996), suggesting that other mycobacterial lipids with PI moieties, such as LAM and PIM, could potentiate the effects of TDM.

Our finding that maturation of TDM bead phagosomes in activated $M \Phi$ is strikingly different from resting $M \Phi$ is in line with studies showing that activation of $M \Phi$ releases the arrest of phagosome maturation by mycobacteria (Schaible et al., 1998; Via et al., 1998). From these studies, however, it remained unclear how activated МФ affected mycobacteria. Targeting effector molecules of activated $M \Phi$ into the intracellular niche of mycobacteria or sequestrating the pathogen from essential nutrients, such as iron, most probably affects mycobacterial growth and therefore interferes with expression/secretion of virulence factors. Thereby, phagosome maturation is directly or indirectly promoted in activated MФ. However, our observation that TDM bead phagosomes quickly matured to phagolysosomes in IFN- $\gamma /$ LPS-activated M $\Phi$ suggests general elevation of phagosomal maturation by upregulation of SNAREs and GTPases, such as VAMP-3 or LRG47/IRGM, respectively (Gutierrez et al., 2004; Murray et al., 2005; Singh et al., 2006), or a direct effect of locally released microbicidals, such as $\mathrm{ROI}$ and $\mathrm{RNI}$ on the lipid.

In this study, we show for the first time that $М \Phi$ activated by IFN- $\gamma /$ LPS specifically inactivate a mycobacterial virulence factor through production of RNI. We found NOS-2 on membranes of TDM bead phagosomes only in fully (IFN- $\gamma /$ LPS) activated MФ. This indicates direct targeting of toxic $\mathrm{RNI}$ including $\mathrm{NO}$ to the phagosomal content. Further reaction with superoxide produced by the NADPH-oxidase may secondarily lead to highly reactive peroxynitrite. Activated NOS-2-deficient or NOS-2inhibited M $\Phi$ failed to interfere with TDM-mediated inhibi- tion of phagosome maturation, while chemical treatment of TDM with NO donors abolished its inhibitory capacity and promoted phagosome maturation even in NOS-2deficient MФ. Taken together, this demonstrates the essential role of RNI during $\mathrm{M} \Phi$ activation for specific and local neutralization of a single virulence factor.

Previously, it was shown that acidification of phagosomes containing live $M$. tuberculosis in activated MФ happened $1 \mathrm{~h}$ after uptake whether NOS-2 was present or not (MacMicking, 2005). However, in comparison with live mycobacteria, our TDM beads revealed the most striking effect on phagosome maturation 30 min after uptake. Their subsequent delivery to phagolysosomes at later time points is probably due to: (i) the limited supply of TDM and (ii) the lack of additional mycobacterial factors including other lipids supplied by live mycobacteria which could contribute to the phagosomal maturation arrest. Inhibition of phagolysosome formation is most probably not a single-factor event, and other glycolipids, such as ManLAM and PIM, may synergize with TDM to block mycobacterial phagosome maturation (Fratti et al., 2003; Vergne et al., 2003; Kang et al., 2005). Such co-factors could be neutralized in an NO-independent manner in activated $\mathrm{M} \Phi$, explaining the reported maturation of mycobacterial phagosomes $1 \mathrm{~h}$ after uptake in nos-2-ko cells (MacMicking et al., 2003).

The NMR analysis revealed that a chemical modification of hydroxyl groups by $\mathrm{NO}$ is responsible for the neutralization of TDM's virulence function. This explains abolishment of TDM-mediated inhibition of phagosome maturation in activated M $\Phi$, where NOS-2 is recruited to the phagosome. This evidences that these hydroxyl groups are essential for the virulence activity of TDM. TDM forms a well-organized phase within monolayers and its molecular packing depends on the interaction between the $\mathrm{OH}$ groups of the mycoloyl residues (Durand et al., 1979). Altering such residues could have a general impact on the functions of TDM. We did not find modifications of double bonds within the mycolic acid chains, which is interesting as NO and peroxynitrite have been shown to break up double bonds within linoleates leading to nitration of the lipid (O'Donnell et al., 1999). This suggests that most of the acyl chains of bead-coupled TDM are protected from RNI/ROI. SIN-1 treatment modified TDM quantitatively. Therefore, activated MФ might be able to completely inactivate all TDM molecules produced by mycobacteria to promote phagosome maturation.

The NO-mediated inactivation of trehalose-associated hydroxyl groups strongly indicates their involvement in inhibition of phagosome maturation. The polar disaccharide residues of TDM could directly interact with polar head groups of membrane phospholipids. This model, which does not require release of TDM and its intercala- 
tion into host cell membranes to inhibit phagosome maturation, is supported by our finding that TDM is not released from the beads in detectable amounts and by recent observations that trehalose alone interacts with the hydrophilic head groups of polar phospholipids, thereby slowing down membrane dynamics, which can affect fusion events required for phagosome maturation (Doxastakis et al., 2005). This suggests that TDM acts on the phagosomal membrane from the surface of the bead in order to delay phagosome maturation rather than by intercalating into the membrane. This may also be indicated by the intimate association between TDM bead surface and phagosome membrane which is abolished upon МФ activation and destruction of TDM's hydroxyl groups by RNI.

Our study provides compelling evidence that the mycobacterial glycolipid TDM is sufficient to retard phagosome maturation at an early endosomal stage in a manner similar to mycobacteria. This qualifies TDM as a key factor in intracellular survival of mycobacteria. Yet other factors may synergize with TDM to generate and maintain the early endosomal niche of $M$. tuberculosis. Like mycobacteria, TDM looses its virulence function in activated M $\Phi$ (Schaible et al., 1998). Loss of function is due to chemical modification of TDM by RNI. Direct exposure of TDM to NO destroyed the glycolipid's hydroxyl groups and normalized phagosome maturation, which identifies these moieties as bearers of TDM's virulence function. Thus, distinct IFN- $\gamma /$ LPS-induced molecules specifically modify an essential virulence factor of the tubercle bacillus, causing enhanced phagolysosome formation and eventually killing of mycobacteria. Inactivation of TDM by activated macrophages is therefore a crucial step in protective immune control of tuberculosis.

\section{Experimental procedures}

\section{Lipids}

The TDM and TMM were isolated from M. bovis BCG and analysed as described before for purity by HPTLC and ${ }^{1} \mathrm{H}-\mathrm{NMR}$ (Fig. S1C and data not shown) (Fischer et al., 2001; 2002). As reference, TDM was purchased from Sigma (Munich, Germany). Lipids were extracted in chloroform/methanol (2:1). One-dimensional HPTLC plates were developed using 40:10:1 chloroform/methanol/ammonia hydroxide or 90:10:6:1 chloroform/methanol/acetone/acetic acid, and stained by spraying with $\alpha$-Naphthol and/or sulfuric acid spray and charring at $200^{\circ} \mathrm{C}$ as described (Fischer et al., 2001; 2002).

\section{Bacterial and cell culture}

Murine wild-type C57BL/6 or NOS-2-deficient M $\Phi$ were isolated from bone marrow, differentiated and maintained in culture as previously described (Schaible and Kaufmann, 2002). RAW-B M $\Phi$ stably expressing PX-GFP were a kind gift from Phillip Hawkins (Cambridge, UK; Ellson et al., 2001) and were cultured in RPMI medium (Gibco) containing 10\% heat-inactivated FCS (Seromed, Germany), $200 \mathrm{mM}$ L-glutamine, $1 \mathrm{mM}$ Hepes, $14.3 \mathrm{mM}$ $\beta$-mercaptoethanol and $0.4 \mathrm{mg} \mathrm{ml}^{-1} \mathrm{G} 418$. MФ were activated for $16 \mathrm{~h}$ with $1000 \mathrm{U} \mathrm{ml}^{-1}$ recombinant mouse IFN- $\gamma$ (R and D Systems) and/or $1 \mathrm{\mu g} \mathrm{ml}^{-1}$ LPS (from Salmonella typhimurium; Sigma) for $4 \mathrm{~h}$ before infection.

Mycobacterium avium-intracellulare strain 101 is a clinical isolate (Schaible et al., 1998). Mycobacteria were labelled with Texas Red as described (Schaible and Kaufmann, 2002). Bacteria were grown in Middlebrook $7 \mathrm{H} 9$ broth supplemented with OADC (Difco) and $10 \mu \mathrm{g} \mathrm{ml}^{-1}$ hygromycin was used for recombinant strains.

\section{Bead preparations}

Tosyl-activated magnetic beads ( $\varnothing 2 \mu \mathrm{m}$; Dynal-Invitrogen, Karlsruhe, Germany) were linked to sterile BSA in PBS (1 h head over). BSA beads $\left(10^{7}\right)$ were coated with lipids after removal of aqueous solvent and mixing with $100 \mu \mathrm{g}$ of lipids (TDM, TMM) disolved in chloroform/methanol (2:1) followed by vapourization of the organic solvents. Dried beads were re-suspended in PBS, sonified for $10 \mathrm{~min}$ and excessively rinsed. HPTLC of extracted lipids before and after coating onto beads revealed equal efficacies of either TMM or TDM coating (Fig. S1C). BSA, TMM as well as TDM beads were phagocytosed in similar numbers by both resting and activated $M \Phi$, indicating similar ways and efficiencies of ingestion for all three particles (data not shown).

\section{Labelling of endocytic compartments}

Early endosomes were revealed by incubating cells with $10 \mu \mathrm{g} \mathrm{ml}^{-1} \mathrm{Tf}-\mathrm{CY} 3$ in Ringer solution $10 \mathrm{~min}$ prior to rinsing and fixation (Schaible and Kaufmann, 2002). Late endosomes and lysosomes were stained by pulsing cells with $50 \mathrm{\mu g} \mathrm{ml}^{-1}$ dexTR (10 kDa; Molecular Probes, Invitrogen, Germany) in medium for $2 \mathrm{~h}$ followed by a $2 \mathrm{~h}$ chase in dye-free medium (Schaible and Kaufmann, 2002).

\section{Phagocytosis assay}

Macrophages were seeded onto $12 \mathrm{~mm}$ round coverslips $\left(10^{5}\right.$ per coverslip). Beads were added at a multiplicity of five beads per cell. Cells were placed on a magnet for $10 \mathrm{~s}$ and subsequently incubated for different time points at $37^{\circ} \mathrm{C}$. Cells were washed with PBS and fixed for $10 \mathrm{~min}$ in $4 \%$ PFA/PBS. For phagocytosis of bacteria, cells were grown in 8-well chamber slides (Nalgene Nunc International, USA). Mycobacteria were added at a multiplicity of infection of 10 and slides were spun at 800 r.p.m. per $4^{\circ} \mathrm{C}$ for $3 \mathrm{~min}$. After incubation for the indicated times at $37^{\circ} \mathrm{C}$, cells were rinsed and fixed in $4 \%$ PFA/PBS for $1 \mathrm{~h}$.

\section{Immunofluorescence and antibodies}

Fixed cells were permeabilized for 5 min with $0.1 \%$ Triton X-100/ PBS. Cells were washed with PBS and incubated for $1 \mathrm{~h}$ at room temperature (RT) with blocking solution (10\% goat serum, $0.05 \%$ Tween/PBS, $0.05 \%$ sodium azide). First and secondary antibodies were diluted $1: 100$ in blocking buffer and added to the cells 
for 30 min. Cells were stained with rat anti-TfR (TIB 219) and rat anti-LAMP1 (ID4B) followed by goat anti-rat antibodies coupled to CY2 or CY3 (Developmental Studies Hybridoma Bank, lowa City, IA, USA; Dianova, Hamburg, Germany). Cells were embedded in Mowiol for analysis by fluorescence or confocal LSM (DMIRBE, Leica, Heidelberg, Germany; LSM 510, Zeiss, Jena, Germany).

\section{Western blot analysis of bead phagosomes}

Bead-containing phagosomes were isolated and purified as previously described and analysed by Western Blot using specific antibodies (for details see Supplementary material) (SturgillKoszycki et al., 1996; Skinner and Wildemann, 1999; Schaible and Kaufmann, 2002).

\section{pH measurement}

For measuring intraphagosomal pH, LysoSensor Yellow/Blue (10 $\mu \mathrm{M}$ in Ringer solution; Molecular Probes) was employed. See Supplementary material for detailed procedure. For each condition, ratios were calculated for 7-20 individual bead phagosomes and differences between groups were assessed by unpaired Student's $t$-test. For each condition, at least three independent experiments were performed.

\section{Nitric-oxide/superoxide treatment of TDM beads}

The TDM-coated beads were incubated with $1 \mathrm{mM}$ of the $\mathrm{NO} / \mathrm{O}_{2}{ }^{-}$ donors SIN-1 or SNP in $1 \mathrm{ml}$ of $\mathrm{H}_{2} \mathrm{O}$ and rotated for $1 \mathrm{~h}$ at RT. Beads were washed five times in water, and lipid was extracted by rotation for $1 \mathrm{~h}$ with $1 \mathrm{ml}$ of chloroform/methanol (2:1). The extracted lipids were weighted and analysed by HPTLC before equal amounts were coated onto fresh BSA beads for phagocytosis assays. HPTLC of treated versus untreated lipids did not reveal differences in migration (data not shown).

\section{Electron microscopy}

Cells were incubated with beads for 10 or $30 \mathrm{~min}$ at $37^{\circ} \mathrm{C}$ before medium was removed and cells were fixed with $2.5 \%$ glutaraldehyde in PBS. Samples were embedded in resin (Schaible et al., 2003). Lead citrate contrasted ultra-thin sections were analysed using a LEO 906E electron microscope equipped with a MORADA digital camera.

\section{Nuclear magnetic resonance}

$1 \mathrm{H}-\mathrm{NMR}$ spectra and $1 \mathrm{H}-13 \mathrm{C}$ shift correlations were recorded with standard pulse sequences on a Bruker $600 \mathrm{MHz}$ NMR spectrometer at $300 \mathrm{~K}$. Both forms of TDM were dissolved in deuterated chloroform/methanol (2:1).

\section{Acknowledgements}

The authors would like to thank Kristine Hagens, Britta Laube and Christian Goosmann for their expert technical help, and Dr Martine Gilleron (Institut de Pharmacologie et de Biologie Structurale,
CNRS, Toulouse, France) for critically reading the manuscript. This work was funded through the German Science Foundation (SPP1130; U.E.S., A.H.) (SFB 670; A.H.), the Royal Society Wolfson Research Merit Award, UK (U.E.S.), and the Wellcome Trust Project Grant 082825/Z/07/Z Mycobacterium tuberculosis lipids in inhibition of phagosome maturation, UK (U.E.S.).

\section{References}

Almog, R., and Mannella, C.A. (1996) Molecular packing of cord factor and its interaction with phosphatidylinositol in mixed monolayers. Biophys J 71: 3311-3319.

Barry, C.E., 3rd, Lee, R.E., Mdluli, K., Sampson, A.E., Schroeder, B.G., Slayden, R.A., and Yuan, Y. (1998) Mycolic acids: structure, biosynthesis and physiological functions. Prog Lipid Res 37: 143-179.

Beatty, W.L., Rhoades, E.R., Ullrich, H.J., Chatterjee, D., Heuser, J.E., and Russell, D.G. (2000) Trafficking and release of mycobacterial lipids from infected macrophages. Traffic 1: 235-247.

de Chastellier, C., and Thilo, L. (1997) Phagosome maturation and fusion with lysosomes in relation to surface property and size of the phagocytic particle. Eur J Cell Biol 74: 49-62.

de Chastellier, C., and Thilo, L. (2006) Cholesterol depletion in Mycobacterium avium-infected macrophages overcomes the block in phagosome maturation and leads to the reversible sequestration of viable mycobacteria in phagolysosome-derived autophagic vacuoles. Cell Microbiol 8: 242-256.

Chua, J., and Deretic, V. (2004) Mycobacterium tuberculosis reprograms waves of phosphatidylinositol 3-phosphate on phagosomal organelles. J Biol Chem 279: 36982-36992.

Clemens, D.L., and Horwitz, M.A. (1995) Characterization of the Mycobacterium tuberculosis phagosome and evidence that phagosomal maturation is inhibited. $J$ Exp Med 181: 257-270.

Clemens, D.L., and Horwitz, M.A. (1996) The Mycobacterium tuberculosis phagosome interacts with early endosomes and is accessible to exogenously administered transferrin. $J$ Exp Med 184: 1349-1355.

Copenhaver, R.H., Sepulveda, E., Armitige, L.Y., Actor, J.K., Wanger, A., Norris, S.J., et al. (2004) A mutant of Mycobacterium tuberculosis $\mathrm{H} 37 \mathrm{Rv}$ that lacks expression of antigen $85 \mathrm{~A}$ is attenuated in mice but retains vaccinogenic potential. Infect Immun 72: 7084-7095.

Crowe, L.M., Spargo, B.J., Ioneda, T., Beaman, B.L., and Crowe, J.H. (1994) Interaction of cord factor (alpha, alpha'trehalose-6,6'-dimycolate) with phospholipids. Biochim Biophys Acta 1194: 53-60.

Doxastakis, M., Sum, A.K., and de Pablo, J.J. (2005) Modulating membrane properties: the effect of trehalose and cholesterol on a phospholipid bilayer. J Phys Chem B Condens Matter Mater Surf Interfaces Biophys 109: 24173-24181.

Durand, E., Welby, M., Laneelle, G., and Tocanne, J.F. (1979) Phase behaviour of cord factor and related bacterial glycolipid toxins. A monolayer study. Eur J Biochem 93: 103-112.

Ellson, C.D., Anderson, K.E., Morgan, G., Chilvers, E.R., Lipp, P., Stephens, L.R., and Hawkins, P.T. (2001) Phos- 
phatidylinositol 3-phosphate is generated in phagosomal membranes. Curr Biol 11: 1631-1635.

Fischer, K., Chatterjee, D., Torrelles, J., Brennan, P.J., Kaufmann, S.H., and Schaible, U.E. (2001) Mycobacterial lysocardiolipin is exported from phagosomes upon cleavage of cardiolipin by a macrophage-derived lysosomal phospholipase A2. J Immunol 167: 2187-2192.

Fischer, K., Collins, H., Taniguchi, M., Kaufmann, S.H., and Schaible, U.E. (2002) IL-4 and T cells are required for the generation of IgG1 isotype antibodies against cardiolipin. J Immunol 168: 2689-2694.

Fratti, R.A., Chua, J., Vergne, I., and Deretic, V. (2003) Mycobacterium tuberculosis glycosylated phosphatidylinositol causes phagosome maturation arrest. Proc Natl Acad Sci USA 100: 5437-5442.

Geisel, R.E., Sakamoto, K., Russell, D.G., and Rhoades, E.R. (2005) In vivo activity of released cell wall lipids of Mycobacterium bovis bacillus Calmette-Guerin is due principally to trehalose mycolates. J Immunol 174: 5007-5015.

Gordon, A.H., Hart, P.D., and Young, M.R. (1980) Ammonia inhibits phagosome-lysosome fusion in macrophages. Nature 286: 79-80.

Gutierrez, M.G., Master, S.S., Singh, S.B., Taylor, G.A., Colombo, M.I., and Deretic, V. (2004) Autophagy is a defense mechanism inhibiting BCG and Mycobacterium tuberculosis survival in infected macrophages. Cell 119: 753-766.

Indrigo, J., Hunter, R.L., Jr and Actor, J.K. (2002) Influence of trehalose 6,6'-dimycolate (TDM) during mycobacterial infection of bone marrow macrophages. Microbiology 148: 1991-1998.

Indrigo, J., Hunter, R.L., Jr and Actor, J.K. (2003) Cord factor trehalose 6,6'-dimycolate (TDM) mediates trafficking events during mycobacterial infection of murine macrophages. Microbiology 149: 2049-2059.

Kang, P.B., Azad, A.K., Torrelles, J.B., Kaufman, T.M., Beharka, A., Tibesar, E., et al. (2005) The human macrophage mannose receptor directs Mycobacterium tuberculosis lipoarabinomannan-mediated phagosome biogenesis. J Exp Med 202: 987-999.

Kaufmann, S.H., and McMichael, A.J. (2005) Annulling a dangerous liaison: vaccination strategies against AIDS and tuberculosis. Nat Med 11: S33-S44.

Kelley, V.A., and Schorey, J.S. (2004) Modulation of cellular phosphatidylinositol 3-phosphate levels in primary macrophages affects heat-killed but not viable Mycobacterium avium's transport through the phagosome maturation process. Cell Microbiol 6: 973-985.

MacMicking, J.D. (2005) Immune control of phagosomal bacteria by p47 GTPases. Curr Opin Microbiol 8: 74-82.

MacMicking, J.D., Taylor, G.A., and McKinney, J.D. (2003) Immune control of tuberculosis by IFN-gamma-inducible LRG-47. Science 302: 654-659.

Martin, E., Nathan, C., and Xie, Q.W. (1994) Role of interferon regulatory factor 1 in induction of nitric oxide synthase. J Exp Med 180: 977-984.

Murray, R.Z., Kay, J.G., Sangermani, D.G., and Stow, J.L. (2005) A role for the phagosome in cytokine secretion. Science 310: 1492-1495.

O'Donnell, V.B., Eiserich, J.P., Chumley, P.H., Jablonsky, M.J., Krishna, N.R., Kirk, M., et al. (1999) Nitration of unsaturated fatty acids by nitric oxide-derived reactive nitrogen species peroxynitrite, nitrous acid, nitrogen dioxide, and nitronium ion. Chem Res Toxicol 12: 8392.

Oh, Y.K., and Straubinger, R.M. (1996) Intracellular fate of Mycobacterium avium: use of dual-label spectrofluorometry to investigate the influence of bacterial viability and opsonization on phagosomal $\mathrm{pH}$ and phagosomelysosome interaction. Infect Immun 64: 319-325.

Purdy, G.E., Owens, R.M., Bennett, L., Russell, D.G., and Butcher, B.A. (2005) Kinetics of phosphatidylinositol-3phosphate acquisition differ between IgG bead-containing phagosomes and Mycobacterium tuberculosis-containing phagosomes. Cell Microbiol 7: 1627-1634.

Rao, V., Gao, F., Chen, B., Jacobs, W.R. Jr and Glickman, M.S. (2006) Trans-cyclopropanation of mycolic acids on trehalose dimycolate suppresses Mycobacterium tuberculosis-induced inflammation and virulence. J Clin Invest 116: 1660-1667.

Russell, D. (2005) Mycobacterium tuberculosis: the indigestible microbe. In Tuberculosis and the Tubercle Bacillus. Cole, S.T., Eisenach, K.D., McMurray, D.N., Jacobs, W.R., Jr (eds). Washington, DC: American Society for Microbiology Press, pp. 427-436.

Russell, D.G., Dant, J., and Sturgill-Koszycki, S. (1996) Mycobacterium avium- and Mycobacterium tuberculosiscontaining vacuoles are dynamic, fusion-competent vesicles that are accessible to glycosphingolipids from the host cell plasmalemma. J Immunol 156: 4764-4773.

Schaible, U.E., and Kaufmann, S.H. (2002) Studying trafficking of intracellular pathogens in antigen presenting cells. In Zychlinski, A., Sansonetti, P. (eds). Methods in Microbiology, Vol. 31, Chapter 19. Academic Press: New York, pp. 343-360.

Schaible, U.E., and Kaufmann, S.H. (2004) Iron and microbial infection. Nat Rev Microbiol 2: 946-953.

Schaible, U.E., Sturgill-Koszycki, S., Schlesinger, P.H., and Russell, D.G. (1998) Cytokine activation leads to acidification and increases maturation of Mycobacterium aviumcontaining phagosomes in murine macrophages. $J$ Immunol 160: 1290-1296.

Schaible, U.E., Winau, F., Sieling, P.A., Fischer, K., Collins, H.L., Hagens, K., et al. (2003) Apoptosis facilitates antigen presentation to T lymphocytes through MHC-I and CD1 in tuberculosis. Nat Med 9: 1039-1046.

Scott, C.C., Botelho, R.J., and Grinstein, S. (2003) Phagosome maturation: a few bugs in the system. J Membr Biol 193: 137-152.

Singh, S.B., Davis, A.S., Taylor, G.A., and Deretic, V. (2006) Human IRGM induces autophagy to eliminate intracellular mycobacteria. Science 313: 1438-1441.

Skinner, M.A., and Wildeman, A.G. (1999) beta (1) integrin binds the $16-\mathrm{kDa}$ subunit of vacuolar $\mathrm{H}(+)$-ATPase at a site important for human papillomavirus E5 and plateletderived growth factor signaling. J Biol Chem 274: 2311923127.

Spargo, B.J., Crowe, L.M., Ioneda, T., Beaman, B.L., and Crowe, J.H. (1991) Cord factor (alpha, alpha-trehalose 6,6'dimycolate) inhibits fusion between phospholipid vesicles. Proc Natl Acad Sci USA 88: 737-740.

Sturgill-Koszycki, S., Schlesinger, P.H., Chakraborty, P., 
Haddix, P.L., Collins, H.L., Fok, A.K., et al. (1994) Lack of acidification in Mycobacterium phagosomes produced by exclusion of the vesicular proton-ATPase. Science 263: 678-681.

Sturgill-Koszycki, S., Schaible, U.E., and Russell, D.G. (1996) Mycobacterium-containing phagosomes are accessible to early endosomes and reflect a transitional state in normal phagosome biogenesis. EMBO J 15: 6960-6968.

Sut, A., Sirugue, S., Sixou, S., Lakhdar-Ghazal, F., Tocanne, J.F., and Laneelle, G. (1990) Mycobacteria glycolipids as potential pathogenicity effectors: alteration of model and natural membranes. Biochemistry 29: 8498-8502.

Vergne, I., Chua, J., and Deretic, V. (2003) Tuberculosis toxin blocking phagosome maturation inhibits a novel $\mathrm{Ca} 2+/$ calmodulin-PI3K hVPS34 cascade. J Exp Med 198: 653659.

Vergne, I., Fratti, R.A., Hill, P.J., Chua, J., Belisle, J., and Deretic, V. (2004) Mycobacterium tuberculosis phagosome maturation arrest: mycobacterial phosphatidylinositol analog phosphatidylinositol mannoside stimulates early endosomal fusion. Mol Biol Cell 15: 751-760.

Vergne, I., Chua, J., Lee, H.H., Lucas, M., Belisle, J., and Deretic, V. (2005) Mechanism of phagolysosome biogenesis block by viable Mycobacterium tuberculosis. Proc Natl Acad Sci USA 102: 4033-4038.

Via, L.E., Fratti, R.A., McFalone, M., Pagan-Ramos, E., Deretic, D., and Deretic, V. (1998) Effects of cytokines on mycobacterial phagosome maturation. J Cell Sci 111 (Part 7): 897-905.

Wagner, D., Maser, J., Lai, B., Cai, Z., Barry, C.E., 3rd, Honer Zu Bentrup, K., et al. (2005) Elemental analysis of Mycobacterium avium-, Mycobacterium tuberculosis-, and Mycobacterium smegmatis-containing phagosomes indicates pathogen-induced microenvironments within the host cell's endosomal system. J Immunol 174: 1491-1500.

Yates, R.M., Hermetter, A., and Russell, D.G. (2005) The kinetics of phagosome maturation as a function of phagosome/lysosome fusion and acquisition of hydrolytic activity. Traffic 6: 413-420.

Yuan, Y., Zhu, Y., Crane, D.D., and Barry, C.E., 3rd. (1998) The effect of oxygenated mycolic acid composition on cell wall function and macrophage growth in Mycobacterium tuberculosis. Mol Microbiol 29: 1449-1458.

\section{Supplementary material}

The following supplementary material is available for this article online:

Fig. S1. A. TDM, but not TMM bead phagosomes retain Tf. Bone marrow derived macrophages (BMM $\Phi)$ were incubated with TMM or TDM beads for 10 min or 30 min. Early endosomes were labelled by a $10 \mathrm{~min}$ Tf-CY3 pulse. Cells were fixed and stained for LAMP-1. Inserts: magnified images of one representative bead phagosome. Bar, $5 \mu \mathrm{m}$.

B. Quantification of Tf and LAMP-1 staining in 100 bead phagosomes showing means \pm standard deviation of three independent experiments.

C. Equal lipid amounts are coupled on beads. HPTLC of TDM and TMM before coating and after re-extraction from coated beads. $D$. The TDM effect is transient. BMM $\Phi$ were incubated with TDM beads for $10 \mathrm{~min}, 30 \mathrm{~min}, 1 \mathrm{~h}$ or $2 \mathrm{~h}$. Lysosomes/late endosomes were labelled by a $2 \mathrm{~h}$ dexTR pulse followed by a $2 \mathrm{~h}$ chase. Alternatively, early endosomes were labelled by Tf-CY3. Cells were fixed and stained for TfR or LAMP1 and visualized using a Cy2-labelled secondary antibody. Shown is the quanitification of 100 bead phagosomes with means \pm standard deviation of three independent experiments.

Fig. S2. Kinetics of BSA bead phagosome maturation in RAW cells expressing PX-GFP.

A. Cells were incubated for 10 or 30 min with BSA beads, fixed, stained for LAMP-1 using a Cy3-labelled secondary antibody and analysed by fluorescence microscopy. DIC, differential interference contrast. Bar: $5 \mu \mathrm{m}$.

B. Quantitative analysis of PX-GFP and LAMP-1 presence (\% colocalization) in BSA bead phagosomes. Approximately 30-40 BSA bead phagosomes were analysed per time. Shown are means \pm standard deviation of three independent experiments. $\mathrm{C}$ and D. TDM beads maintain $\mathrm{PI}(3) \mathrm{P}$ on phagosomes for $1 \mathrm{~h}$. RAW M $\Phi$ expressing PX-GFP were incubated with Alexa568labelled $M$. tuberculosis $(C)$ or $M$. bovis BCG expressing DsRed (D) for 1, 4 and $24 \mathrm{~h}$. Cells were fixed, analysed by confocal microscopy. Both mycobacterial species were found in PX-GFPpositive phagosomes $1 \mathrm{~h} \mathrm{p}$.i. but, already $4 \mathrm{~h}$ p.i., more than $60 \%$ of the phagosomes and, $24 \mathrm{~h}$ p.i., all mycobacterial phagosome became negative for PX-GFP. This indicates that, although $\mathrm{PI}(3) \mathrm{P}$ synthesis is maintained for longer on phagosomes containing mycobacteria when compared with those containing normal beads, it is a decelerated yet temporary event in the fate of a mycobacterium's phagosome. Arrowheads indicate PXGFP-positive phagosomes.

Fig. S3. A. Purified TDM bead phagosomes contain predominantly early but less late endosomal markers. Western blot of TMM and TDM bead phagosomes purified from cells $30 \mathrm{~min}$ after uptake. Equal amount of protein was loaded $(10 \mu \mathrm{g})$. Lysates from the same cells served to control for equal expression of the marker proteins. Blots were stained using antibodies to the indicated proteins and binding signals were detected using HRPlabelled secondary antibodies and the ECL system. $\mathrm{M}=\mathrm{TMM}$; $\mathrm{D}=$ TDM.

B. Densitometric quantification of the Western blot shown in A. C. TDM bead phagosomes contain less lysosomal enzyme activity. Enzyme activities of purified TDM and TMM bead phagosomes in comparison with whole-cell lysates were analysed for the lysosomal enzymes $\beta$-hexosaminidase and $\beta$-galactosidase using specific fluorescent substrates.

D. BSA bead phagosomes acidify between 8 and 18 min after uptake. ВMM $\Phi$ were incubated with BSA beads in the presence of $15 \mu \mathrm{M}$ LysoSensor Yellow/Blue for 8 or $18 \mathrm{~min}$ and chased for $8 \mathrm{~min}$. Live cells were analysed by fluorescence microscopy using dual excitation and emission filters. Acidified compartments appear in green, non-acidified ones in red. Arrowheads indicate bead phagosomes. For quantification see Fig. 2B. Bar: $10 \mu \mathrm{m}$. Fig. S4. Phagosomes containing TDM beads mature in

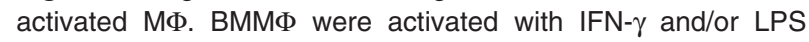
and incubated with TDM beads. Early endosomes (A) or late endosomes/lysosomes (B) were detected with Tf-CY3 or dexTR respectively. After fixation, cells were co-stained for TfR $(B)$ or LAMP-1 (A) and analysed by fluorescence microscopy. For quantification see Fig. 3D. Bar: $10 \mu \mathrm{m}$.

C. Western blot analysis of purified bead phagosomes reveals that TDM bead phagosomes mature in activated $М \Phi$. ВММ $\Phi$ were activated with IFN- $\gamma$ and/or LPS and incubated with TDM 
beads. Subsequently, cells were incubated for 30 min and phagosomes were purified according to Experimental procedures. Equal amounts of proteins $(5 \mu \mathrm{g})$ were loaded. The blot was stained using antibodies for the indicated marker proteins and binding signals were detected using fluorescent secondary antibodies.

Fig. S5. NOS-2 interferes with TDM-mediated inhibition of phagosome maturation.

$\mathrm{A}$ and B. ВММФ from nos-2-ko mice were activated with IFN- $\gamma$ and/or LPS and incubated with TDM beads. Early endosomes (A) or late endosomes/lysosomes (B) were visualized with Tf-CY3 or dexTR respectively. After fixation, cells were co-stained for TfR (B) or LAMP-1 (A) and analysed by fluorescence microscopy.

C. Quantification of Tf-CY3, TfR, dexTR and LAMP-1 colocalization with TDM bead phagosomes in activated and non-activated nos-2-ko МФ. Shown are means \pm standard deviation of three independent experiments; in each experiment, 100 bead phagosomes were analysed.

D. Activated ВМM $\Phi$ from nos-2-ko mice were incubated with TDM beads in the presence of $15 \mu \mathrm{M}$ LysoSensor Yellow/Blue for $8 \mathrm{~min}$ or $18 \mathrm{~min}$ and chased for $8 \mathrm{~min}$. Live cells were analysed by fluorescence microscopy using dual excitation and emission filters. Fluorescent micrographs were recorded at $\mathrm{Ex}_{360 \mathrm{~nm}} / \mathrm{Em}_{445 \mathrm{~nm}}$ (blue) and $E x_{380 \mathrm{~nm}} / \mathrm{Em}_{560 \mathrm{~nm}}$ (green). For quantification see Fig. 4D. Inserts: magnified images of one representative bead phagosome. Bars: $10 \mu \mathrm{m}$.

Fig. S6. A. Inhibition of NOS-2 abolishes interference of TDMmediated inhibition of phagosome maturation in activated $M \Phi$. RAW MФ expressing PX-GFP were either kept resting or acti- vated using IFN- $\gamma$ and LPS overnight. Cells were incubated with L-NAME (Sigma, Munich, Germany; $5 \mu \mathrm{g} \mathrm{ml}^{-1}$ ), a specific NOS-2 inhibitor, for $2 \mathrm{~h}$ before TDM beads were added for $30 \mathrm{~min}$. Cells were fixed and stained for LAMP-1. Arrows indicate bead phagosomes. Inserts: magnified images of few representative bead phagosome. Bars: $10 \mu \mathrm{m}$.

B. Quantification of PI(3)P (PX-GFP) and LAMP-1 staining of TDM bead phagosomes in PX-GFP expressing RAW M $\Phi$, either resting or IFN- $\gamma /$ LPS-activated and subsequently either mock- or L-NAME-treated. TDM beads were added for 10 or $30 \mathrm{~min}$. Shown are means of 100 analysed phagosomes \pm standard deviation of two independent experiments.

Fig. S7. SIN-1 treatment annihilates TDM-mediated inhibition of phagosome acidification. ВМM $\Phi$ from either wild-type mice or nos-2-ko mice were activated as indicated and incubated with TDM beads or beads coated with SIN-1-treated TDM in the presence of $15 \mu \mathrm{M}$ LysoSensor Yellow/Blue for 8 or $18 \mathrm{~min}$ and chased for $8 \mathrm{~min}$. Live cells were analysed by fluorescence microscopy using dual excitation and emission filters. Fluorescent micrographs were recorded at $\mathrm{Ex}_{360 \mathrm{~nm}} / \mathrm{Em}_{445 \mathrm{~nm}}$ (blue) and $\mathrm{Ex}_{380 \mathrm{~nm}} / \mathrm{Em}_{560 \mathrm{~nm}}$ (green). For quantification see Fig. 4E. Inserts: magnified images of few.

This material is available as part of the online article from: http://www.blackwell-synergy.com/doi/abs/10.1111/j.1462-5822. 2008.01147.x

Please note: Blackwell Publishing is not responsible for the content or functionality of any supplementary materials supplied by the authors. Any queries (other than missing material) should be directed to the corresponding author for the article. 